\title{
Wind-Structure System Modeling Based on Multi Material ALE Method
}

\author{
Wang Yang ${ }^{1,2, a}$, Xingman Mo ${ }^{1, b}$, Jian Yang ${ }^{1,2, c}$, Huixin Wang ${ }^{1, d}$, Shunyu Yao ${ }^{1, d}$, \\ Kerui Wang ${ }^{1, \mathrm{~d}}$ \\ ${ }^{1}$ College of Mechanical Engineering, Guangxi University, Nanning 530004, China \\ ${ }^{2}$ Collaborative innovation center for sugar industry of Guangxi, Nanning 530004, China \\ ªyanghope@163.com, ${ }^{\text {b3754499997@qq.com, }, \text { yangokok@gxu.edu.cn, }{ }^{\text {d}} 553914627 @ q q . c o m}$
}

Keywords: multi material ALE, wind - structure system, fluid-structure interaction, simulation

\begin{abstract}
Currently,the process of using multi material ALE method to modeling wind-structure system is not specific ,and its reliability is unknown. This paper details the process of using multi material ALE method to modeling the wind-structure system,and uses the method to model a wind-plate system. Physical verification test and variation analysis of flow field also has been done. The results show that, when the wind speed are $3.46 \mathrm{~m} / \mathrm{s}$ and $4.58 \mathrm{~m} / \mathrm{s}$, the maximum relative error between simulation values and experimental value are $8.6 \%$ and $5.5 \%$, the maximum relative error between simulation values and calculated values are $8.7 \%$ and $5.2 \%$. This shows that we can get high precision of wind-structure system by using multi material ALE method, and it can be applied to the simulation of wind-structure system. This paper provides a basis for using the multi material ALE method to model a wind-sugar cane-soil coupling system.
\end{abstract}

\section{Introduction}

Wind is a natural factor of major influencing of the growth of corps in agricultural production. Especially typhoon, it can lead to crops lodging, broken and uproot which have impacts on its production seriously. Therefore, the study on mechanisms of interaction of typhoon, crops and soils have great importance. However, the new research report about this aspect at home and abroad is rarely at present. And action system between the typhoon, crops and soils are extremely complex fluid-solid coupled system. Therefore, the study on its mechanism must adopt the mean of fluid-solid coupling numerical simulation.

At present, more mature fluid-solid coupling numerical simulation commercial software including: LS-DYNA、ADINA and other software. Among them, LS-DYNA can conduct research on its mechanism by the numerical simulation model of coupling system of typhoon - crop and soil which established by the mean of finite element method of multi - material ALE because of its characteristics of finite element method which can solve multi - material ALE effectively and Pre-Post processing modules of rich material libraries. Firstly, we have to clarify creation method of the coupling numerical simulation model as the establishment of coupling numerical simulation model of typhoon, crops and soils are fairly complex.

At present, research of some scholars at home and abroad are lack of specific descriptions of modeling process structural coupling system about wind [1-3]and their results without precision validation and reliability, though they conducted the related study on the problem of fluid-solid interaction about wind by the method of finite element method of multi - material ALE.

Therefore, this article to establish coupling numerical simulation model of wind - steel plate and conduct physical verification about it by the way of finite element method of multi - material ALE, in order to build the process of structural coupling system model of wind, so that to provide a scientific evidence for the establishment of the model of coupling system of typhoon, crops and soils by the mean of multi - material ALE. 


\section{Establishment of numerical simulation model}

Geometric Model. The numerical simulation model of wind-steel plate system including three components: the source of wind, airshed and steel plate. The scale of source of wind and airshed shall larger than the steel plate properly, and they shall be conode, in order to avoid the influence of wall stress wave on the calculated result and for the exchange of substance and transportation of wind and air when to build the geometric model.

Therefore, to meet the requirements of modeling above and save the time of computing so that to conduct verification of the physical experiment that the component of the source of wind was built in a cubic. The size of its length, width and height are $800 \mathrm{~mm} \times 700 \mathrm{~mm} \times 5 \mathrm{~mm}$, and the airshed also was built in a cubic and its size are $800 \mathrm{~mm} \times 700 \mathrm{~mm} \times 550 \mathrm{~mm}$.

The steel plate is rectangular steel plate, and its center and center of airshed are symmetrical. Its length, width and height are $300 \mathrm{~mm} \times 200 \mathrm{~mm} \times 10 \mathrm{~mm}$.

Meshing and Loading. In this article, the source of wind was divided into one unit to save the time of computing. It demands the size of finite element grid of source of wind and airshed are consistent when simulate wind - structure system by the finite element method of multi - material ALE of LS-DYNA.To conduct grid division of sources of wind and airshed by the method of grid division. The wind source is 2538 units,and the airshed is 98982 units. The grid size of steel plate is similar to airshed,and the steel division number is 560 that is in order to ensure the coupling effect of wind and steel plate.The boundary of airshed defined as non - reflective boundary of the domain in order to simulate the effect of infinite airshed and to avoid wall stress wave on the calculation results.The center of the back of the plate is full degree of freedom constraint to prevent the steel plate blow away by the wind.To formulate the velocity of the wind source into $V$ by imposing the same speed $V$ for the node group of two layers of upper and down of wind source.The finite element model of wind - steel plate system as shown in figure 1,and its model of boundary grid of flow distribution as shown in figure 2.
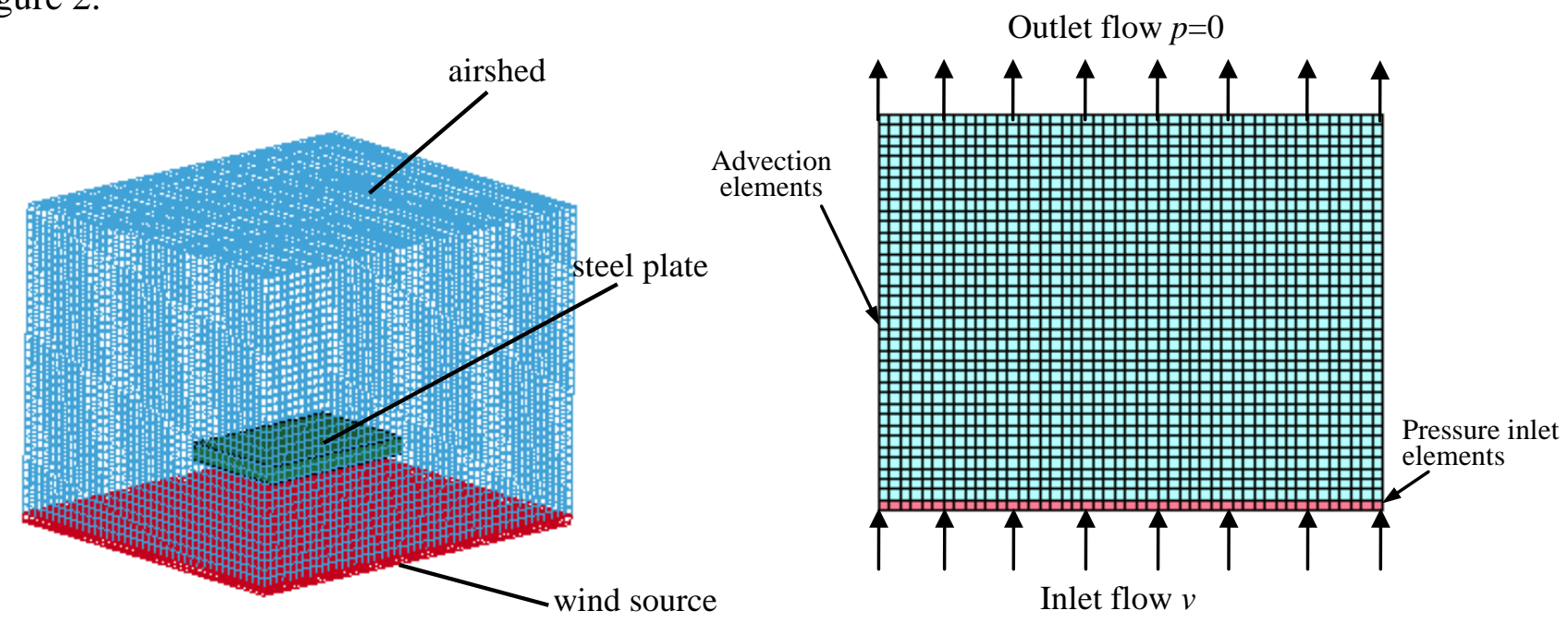

Fig.1 Finite element model of wind-plate system

Fig.2 Fluid boundary mesh model of wind-plate

Material Model. Wind source, airshed and steel plates, to discrete with hexahedron element SOLID 164. To define wind source, airshed as blank material (*MAT_NULL). Its density is $1.29 \mathrm{~kg} / \mathrm{m}^{3}$; cutoff pressure is $-1 \mathrm{~Pa}$, dynamic viscosity coefficient is $1.7456 \times 10^{-5} \mathrm{~Pa} \cdot \mathrm{s}$; specific heat ratio is $\gamma=1.4[4]$. State equation of wind source and airshed adopted Linear Polynomial (*EOS_LINEAR_POLYNOMIAL). The value of its initial energy of $\mathrm{E}_{0}$ per unit volume and Initial relative volume $\mathrm{V}_{0}$ are 253312.5 and 1.0 respectively. Then initial pressure within wind source and airshed are standard atmospheric pressure [4]. To define the steel plate as elastoplastic material, and its density is $7.85 \times 10^{3} \mathrm{~kg} / \mathrm{m}^{3}$; Poisson's ratio is 0.3 ; elasticity modulus is $2.07 \times 10^{11} \mathrm{~Pa}$.

Main control parameters. Control parameter settings have an important influence on accuracy of simulation results. This article set the value of parameter PREF of CONTROL_ALE to $1.01325 \times 10^{5}$,to set the parameter ELFORM of SECTION_SOLID_ALE to 11 (algorithm of multi - 
material ALE),to set parameter AET of solid element algorithm of wind source to 4 (including unit), in order to simulate the actual state of wind source and airshed more accuracy.Meanwhile, to achieve the exchange of substance and transportation between wind source and airshed with keywords ALE_MULTI-MATERIAL_GROUP, and to define hourglass of material and bulk viscosity. The coupling of wind source and steel plate adopted the method of penalty function, and set its number of integral points of coupling to 4 , to reduce leakage. To exert gravity field on the entire model.

\section{Verification of Numerical Simulation Model}

Verification Method and Equipment. The verification of numerical simulation model of system of wind source and steel plate adopted method of physical testing. Verification test used equipment is: Microcomputer control electron universal testing machine (model:NKK-4005,Shenzhen South Instrument \& Equipment Co. Ltd. resolution ratio of force transducer: $0.01 \mathrm{~N}$ ); blower(model: FB-40P, impeller diameter: 400mm); rectangular steel plate(length300mm,width $200 \mathrm{~mm}$, height $10 \mathrm{~mm}$ and with smooth surface). To weld a piece of iron pillar on center of its back for $450 \mathrm{~mm}$ long, diameter of $18 \mathrm{~mm}$ in order to facilitate the fixation. Verification adopted 2 types of wind speed. Diagram of verification testing system was as shown in figure 3.

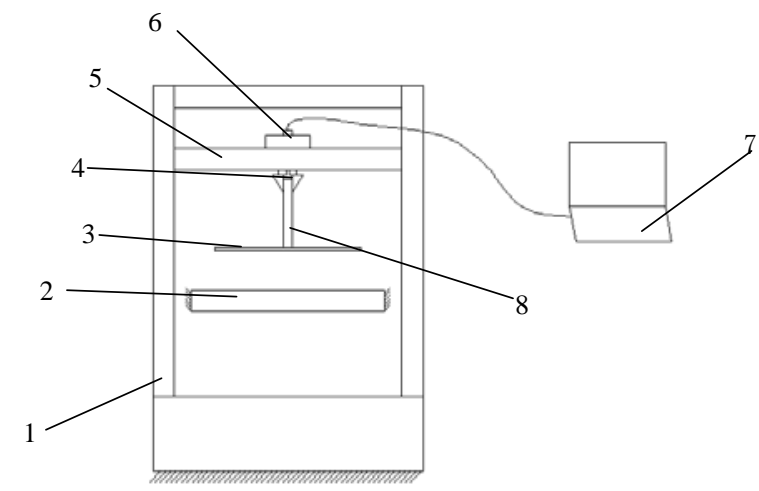

1 frame of universal testing machine 2 blower 3 rectangular steel plate 4 chuck 5 movable cross-beam 6 sensor 7 computer 8 chart of iron pilla

Fig.3 Diagram of verification testing system

Validation results. Fig.4 is curve of force of steel plate under the action of the wind. Set of curves 1(dashes, dots, lines, solid line represent the theoretical values, simulation values and experimental values respectively) have a velocity of $4.58 \mathrm{~m} / \mathrm{s}$. Set of curves 2 (dashes, dots, lines, solid line represents the theoretical values, simulation values and experimental values respectively) with a velocity of $3.46 \mathrm{~m} / \mathrm{s}$. Its theoretical value can be deriving from the product of wind pressure and the windward area of calculation formula [5] of wind pressure. The figure 4 shows that maximum relative error of theoretical calculating values and simulation values are 8.7\%, 5.2\% respectively. Maximum relative error of experimental value and simulation value is $8.6 \%, 5.5 \%$ respectively when the wind speed is $3.46 \mathrm{~m} / \mathrm{s}$ and $4.58 \mathrm{~m} / \mathrm{s}$ respectively. It shows that the numerical simulation model of wind-steel plate system with higher accuracy and the error gets smaller and accuracy will increase with the increase of wind speed. 


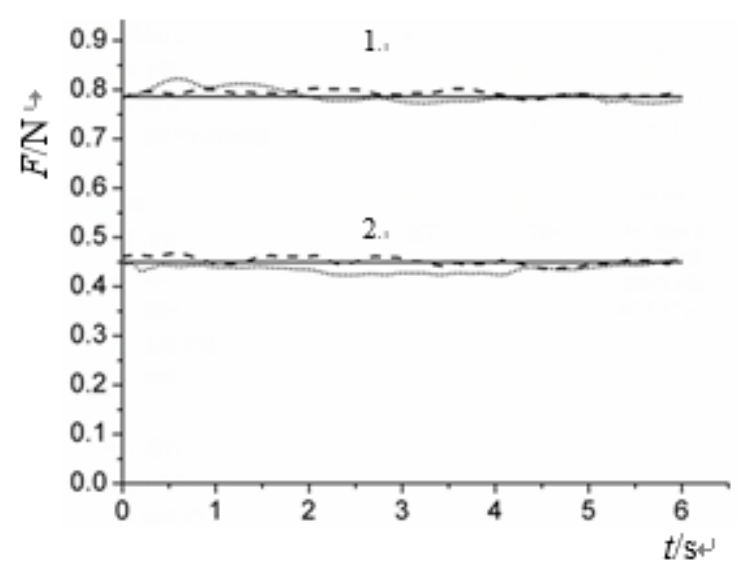

Fig.4 Wind force on the steel plate

\section{Conclusion}

This article adopted the method of comparison between simulation testing, physical testing and theoretical calculation in order to discuss the feasibility to build wind structural numerical simulation model of the coupling system by using finite element method of multi - material ALE. The results show that maximum relative error of simulation value, experimental value and theoretical calculating value are $8.6 \%, 8.7 \%$ respectively, and the relative error will reduce with the increase of wind speed. To build a system model of wind-steel plate by using finite element method of multi - material ALE of LS-DYNA will have a higher accuracy, and it can also use for the process of wind structural modeling, to provide scientific evidence for the modeling of coupling system of typhoon - crops soils by the method of finite element of multi - material ALE.

\section{Acknowledgment}

This work was supported by a grant from the Guangxi Natural Science Foundation (Grant No.2014GXNSFBA118279), College Scientific Research Foundation of Guangxi Education Department (Grant No.YB2014002), Scientific Research Foundation of Guangxi University (Grant No.XJZ130354) and College Students' Experimental Skills and Innovation Ability Training Foundation of Guangxi University(Grant No.SYJN20130104).

\section{References}

[1] X.M. Zhang, X.L. Jin, X.D. Chen, Study on simulation method of dynamic response of large flexible liquid storage structures under the action of wind load, Vibration and Shock.(2009)115-118.

[2] H.Y. Chen, The Influence of human - javelin systems on initial condition of the flight of the javelin and its computer simulation, Taiyuan University of Technology, Taiyuan, 2007.

[3] C.S. Ma, S.L. Huang, J.H. Zhang, et al, Application of simulation of capsule landing by the method of ALE in LS-DYNA, Journal of Tsinghua University. 48(2006)1455-1457.

[4] H.O. Zhao, Dynamic analysis guide of LS-DYNA, The Publishing House of Ordnance Industry, Beijing, 2003.

[5] X.T. Zhang, Wind-induced vibration calculation and structural wind pressure, Tongji University Press Shanghai, 1985. 